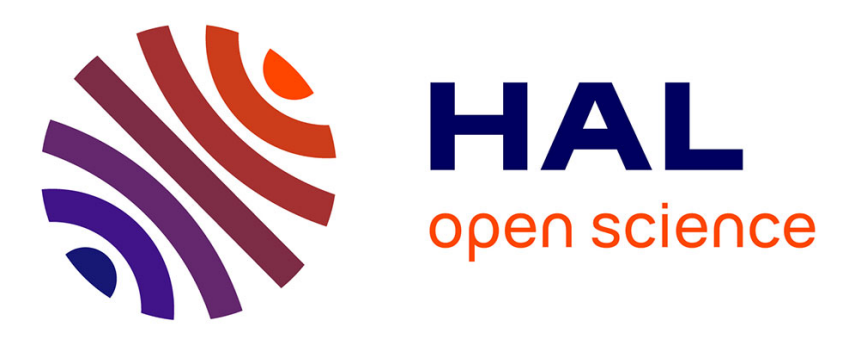

\title{
High-fidelity Sliding-mode Control of a Pneumatic Haptic Teleoperation System
}

\author{
Sean Hodgson, Mahdi Tavakoli, Arnaud Lelevé, Minh Tu Pham
}

\section{To cite this version:}

Sean Hodgson, Mahdi Tavakoli, Arnaud Lelevé, Minh Tu Pham. High-fidelity Sliding-mode Control of a Pneumatic Haptic Teleoperation System. Advanced Robotics, 2014, 28 (10), pp.659-671. 10.1080/01691864.2014.888130 . hal-00926552

\section{HAL Id: hal-00926552 https://hal.science/hal-00926552}

Submitted on 28 Jan 2016

HAL is a multi-disciplinary open access archive for the deposit and dissemination of scientific research documents, whether they are published or not. The documents may come from teaching and research institutions in France or abroad, or from public or private research centers.
L'archive ouverte pluridisciplinaire HAL, est destinée au dépôt et à la diffusion de documents scientifiques de niveau recherche, publiés ou non, émanant des établissements d'enseignement et de recherche français ou étrangers, des laboratoires publics ou privés. 


\title{
High-fidelity sliding-mode control of a pneumatic haptic teleoperation system
}

\author{
Sean Hodgson ${ }^{1}, \quad$ Mahdi Tavakoli ${ }^{1}, \quad$ Arnaud Lelevé ${ }^{2} \quad$ and Minh Tu Pham² \\ ${ }^{1}$ Department of Electrical and Computer Engineering, University of Alberta, Edmonton, AB Canada T6G2V4, \\ sean.hodgson1@gmail.com,mahdi.tavakoli@ualberta.ca \\ ${ }^{2}$ Laboratoire Ampère, UMR CNRS 5005, Université de Lyon, INSA-LYON, F-69621 Villeurbanne Cedex, \\ France, arnaud.leleve@insa-lyon.fr,minh-tu.pham@insa-lyon.fr
}

\begin{abstract}
For a pneumatic teleoperation system with on/off solenoid valves, sliding-mode control laws for position and force, ensuring low switching (open/close) activity of the valves are developed. Since each pneumatic actuator has two pneumatic chambers with a total of four on/off valves, sixteen possible combinations ("operating modes") for the valves' on/off positions exist, but only seven of which are both functional and unique. While previous work has focused on 3-mode slidingbased position control of one pneumatic actuator, this paper develops the seven-mode sliding-based bilateral control of a teleoperation system comprising a pair of pneumatic actuators. The proposed bilateral sliding control schemes are experimentally validated on a pair of actuators utilizing positionposition and force-position teleoperation architectures. The results demonstrate that leveraging the additional modes of operation leads to more efficient and smoother control of the pneumatic teleoperation system. It was found that viscous friction forces were crippling haptic feedback in the position-position architecture. Through the use of force sensors, the force-position architecture was able to compensate for the heavy viscous friction forces.
\end{abstract}

keywords: Pneumatic actuator, on/off solenoid valve, teleoperation, transparency, sliding-mode control

\section{INTRODUCTION}

Teleoperation aims to allow a human operator to carry out a sensing or manipulation task in an environment that is not amenable to direct interaction. The interaction between the human, the teleoperator, and the environment need to be controlled in such a way as to ensure a high level of "fidelity" defined as the accurate transmissions of the environment's mechanical properties to the human operator $[8,9,10,24]$. Bilateral teleoperation systems have been developed for a variety of applications ranging from telesurgery to space exploration $[2,13,18,19,24]$. 
In this paper, we investigate the performance of low-cost pneumatic components when used as actuators in teleoperation systems. Pneumatic actuators offer many advantages for positioning applications such as low maintenance cost, high ratio of power to weight, cleanliness, and safety. Also, where it is not possible to use electric motors such as in robot-assisted surgery under MRI guidance, it is possible to use pneumatic actuators to drive robots. On the down side, pneumatic actuators typically suffer from stiction and sensitivity of the actuator dynamics to external loading and piston position along the cylinder stroke [5]. Thus, designing an effective control architecture for a pneumatic actuator is fairly challenging and this is exacerbated by the nonlinear pressure dynamics in pneumatic chambers [25].

Some pneumatic system utilize servo valves, which allow for a continuous change of the input mass flow rate [14]. However, servo-valves are expensive devices. A low-cost substitute to the servo valve is the on/off (i.e., open/close) solenoid valve, which does not require the expensive components used in manufacturing a servo-valve. On the down side, due to the discrete-input nature of the on/off solenoid valve, it cannot use continuous control laws as was the case with the servo valve. The input discontinuity makes the nonlinear dynamics of the pneumatic actuator harder to control. The noise they produce when actuated by a switching controler may also be another drawback.

Past research has studied using solenoid-valve pneumatic actuators with a pulse width modulated (PWM) input $[1,15,21,23]$. In fact, a PWM input with a sufficiently high frequency to a solenoid valve can approximate the continuous input properties of a servo valve [20].

When considering a single pneumatic actuator that is comprised of two chambers and four on/off solenoid valves, one would assume that there would be a total of sixteen distinct combinations of valves' on or off positions ("operating modes"). Each chamber has two solenoid valves, one that can connect to a supply pressure, and one that can connect to an exhaust pressure. We do not want to connect the chamber to both pressure and exhaust at the same time, therefore we find that seven of the sixteen possible valve combinations are invalid. Of the remaining nine modes, three can be considered functionally equivalent under no-load conditions. Thus, the system has a total of seven unique discrete modes. This point is elaborated in Section 2.

In [16], a sliding-mode control based on three of these discrete modes was applied to a two-chamber solenoid-valve actuator. It demonstrated good tracking and relatively low steady-state position errors. One of the aspects that could be also analyzed while using solenoid valves is the valve switching activity which is defined by the total count of switches performed by all the solenoid valves divided by the total time of use. In [7], it has been demonstrated that for a single pneumatic actuator, expanding the control possibilities from 3-mode control to seven-mode control reduces the tracking error and the valves' switching activity, causing an overall improvement in the pneumatic system performance. The use of such actuation and control in a teleoperation scheme has been studied in [13] but only with a 3-mode control. In this paper, we design a seven-mode based sliding control for application to position-position and force-position control of a teleoperation system. It is expected that the four additional modes of operation help to utilize just the necessary amounts of drive energy, allowing smoother teleoperation control. To assess this conjecture, we compare in experimentations teleoperation performance under 
3-mode sliding control versus 7-mode control.

The organization of this paper is as follows. The discrete-input mode-based model of a single pneumatic actuator with solenoid valves is given in Section 2. Sliding-mode control of a teleoperated pair of pneumatic actuators is reported in Section 3 at first for a position-position scheme and then for a force-position scheme. The experimental results are reported in Section 4 with the the analysis of the

results in Section 5. Finally, the concluding remarks and directions for future research are presented in Section 6.

\section{DISCRETE-INPUT MODEL}

We consider a pneumatic actuator comprised of two chambers as shown in Figure 1. Each chamber is connected via its two solenoid valves to either the supply pressure or the exhaust pressure. The model presented in this section is derived from the one in [7]. It is summed up here as it is necessary for the comprehension of the rest of this paper.

To describe the air flow dynamics in a cylinder, we assume that:

- Air is an ideal gas and its kinetic energy is negligible in the chambers,

- The pressure and the temperature are homogeneous in each chamber,

- The evolution of the gas in each chamber is polytropic,

- The mass flow rate leakages are negligible, and

- The supply and exhaust pressures are constant.

The pressure dynamics of the two chambers of the actuator can be approximated [11] as

$$
\begin{array}{r}
\dot{P}_{P}=\frac{k}{V_{P}}\left(r T_{P} Q_{P}-A_{P} P_{P} \dot{y}\right) \\
\dot{P}_{N}=\frac{k}{V_{N}}\left(r T_{N} Q_{N}+A_{N} P_{N} \dot{y}\right)
\end{array}
$$

where $Q_{P}$ and $Q_{N}$ refer to mass flow rates $(\mathrm{kg} / \mathrm{s})$ of the chambers $\mathrm{P}$ and $\mathrm{N}$, respectively; $T_{N}$ and $T_{P}$ refer to the temperature $(\mathrm{K})$ in chambers $\mathrm{N}$ and $\mathrm{P}$, respectively. In addition, $V_{P}$ and $V_{N}$ refer to volumes $\left(\mathrm{m}^{3}\right)$ of the chambers $\mathrm{P}$ and $\mathrm{N}$, respectively, as shown below:

$$
V_{P}=A_{P}(l / 2+y) \quad V_{N}=A_{N}(l / 2-y)
$$

The mass flow rates $Q_{P}$ and $Q_{N}$ can be derived in terms of the discrete voltage inputs $U_{1}, U_{2}, U_{3}$, and $U_{4}$ and the continuous pressure inputs $P_{P}$ and $P_{N}$.

$$
\begin{gathered}
Q_{P}=U_{1} Q\left(P_{S}, P_{P}\right)-U_{2} Q\left(P_{P}, P_{E}\right) \\
Q_{N}=U_{3} Q\left(P_{S}, P_{N}\right)-U_{4} Q\left(P_{N}, P_{E}\right)
\end{gathered}
$$


As shown below, the model for the mass flow rate has two parameters: the critical pressure ratio $b_{\text {crit }}$ and the mass flow rate constant $C_{\text {val }}=C \rho_{0}$ where $C$ is the sonic conductance $\left(\mathrm{m}^{3} /(\mathrm{s} \mathrm{Pa})\right)$ and $\rho_{0}$ is the density of air $\left(\mathrm{kg} / \mathrm{m}^{3}\right)$ at a reference condition $T_{0}=293 \mathrm{~K}[4]$ :

$$
\begin{aligned}
& Q\left(P_{U p}, P_{\text {Down }}\right)=C_{v a l} P_{U p} \sqrt{\frac{T_{A t m}}{T_{U p}}} \times \\
& \left\{\begin{array}{cl}
\sqrt{1-\left(\frac{\frac{P_{\text {Down }}}{P_{U p}}-b_{\text {crit }}}{1-b_{\text {crit }}}\right)^{2}} & , \text { if } \frac{P_{\text {Down }}}{P_{U p}}>b_{\text {crit }} \text { (subsonic) } \\
1 & \text {,if } \frac{P_{\text {Down }}}{P_{U p}} \leq b_{\text {crit }} \text { (choked) }
\end{array}\right.
\end{aligned}
$$

The value for $b_{\text {crit }}=0.433$ comes directly from the data sheet of our solenoid valves, provided by MatrixBibus (BIBUS France S.A.S, Chaponnay, France). In the above, $T_{U p}$ is the upstream temperature of air and $T_{A t m}$ is the atmospheric temperature.

It may seem that the four on/off solenoid valves of the actuator allow a total of sixteen possible combinations ( "modes") in terms of the valves' on/off positions. However, from a functional perspective, we cannot allow a single chamber to both vent and pressurize at the same time, limiting the actuation possibilities to nine modes. In fact, as far as each chamber of the pneumatic actuator is concerned, its two on/off valves allow it to be in one of three "states": connected to an air supply (pressurizing), connected to exhaust pressure (venting), or closed. Since each chamber is capable of being in one of the above-mentioned three states, the pneumatic actuator with two chambers can have a total of nine discrete modes.

This reflects the maximum number of modes available in a two-chamber pneumatic actuator, meaning that a controller can utilize only a subset of these modes. In general, the usage of more modes by the controller is expected to facilitate the use of more optimal amounts of drive energy, meaning that improved positioning precision and reduced valve switching (open/close) activity should result. This paper studies this issue in the context of teleoperation control of a pair of pneumatic actuators equipped with solenoid valves.

In [16], three out of nine discrete modes have been considered for control of the two-chamber solenoidvalve actuator of Figure 1. In [12], the 3-mode model was expanded to a five-mode model. The 5-mode control has two extra modes that utilize the option to have one chamber closed and the other chamber pressurized. In [6], the five-mode model was further expanded to a new seven-mode model utilizing two new modes. We mentioned previously that there are a total of nine discrete modes for the solenoid valves. The question is whether there is any significant advantage in further expansion of control possibilities from seven to the full nine modes. The nine possible modes are shown in Table 1. refers to an open valve. Reviewing these different discrete modes, we observe that the modes $M_{1}, M_{8}$, and $M_{9}$ are functionally similar (under no load) as they correspond to the two chambers being both closed, both venting, and both pressurized, respectively. For all of these three modes, the pressure difference across the chamber $\mathrm{P}$ and chamber $\mathrm{N}$ is reduced to zero over time, implying that the piston acceleration profile will be similar for them. In our research, the mode $M_{1}$ was selected out of the three equivalent modes $\left(M_{1}\right.$, 
$M_{8}$, and $M_{9}$ ) because it provides the highest resistance to piston motion, which will facilitate reducing the position tracking error to zero. Thus, our modeling and control analysis from this point forward will focus on the modes $M_{1}$ to $M_{7}$.

The dynamics of the pneumatic actuator of Figure 1 involving the chamber pressures and the resulting piston motion is

$$
\left(A_{P} P_{P}-A_{N} P_{N}\right)-b_{V} \dot{y}-\tau_{S t}+\tau_{E x t}=M \ddot{y}
$$

where $P_{P}$ and $P_{N}$ refer to pressures $(\mathrm{Pa})$ inside the chambers $\mathrm{P}$ and $\mathrm{N}$, respectively; $A_{P}$ and $A_{N}$ refer to the piston cylinder areas $\left(\mathrm{m}^{2}\right)$ in the chambers $\mathrm{P}$ and $\mathrm{N}$, respectively; $b_{V}$ is the viscosity coefficient $(\mathrm{N} \mathrm{s} / \mathrm{m}), M$ is the total mass of the load and the piston $(\mathrm{kg}), \tau_{S t}$ is the stiction force $(\mathrm{N}), \tau_{E x t}$ is the external force $(\mathrm{N})$, and $y$ refers to the piston position $(\mathrm{m})$ shown in Figure 1. Note the arrows for position $y$ and force $F_{E x t}$ and $F_{s t}$ in Figure 1 refer to their positive directions. The stiction force $\tau_{S t}$ was considered to be negligible since the pneumatic actuator used in our experiments was an Airpel anti-stiction cylinder (All Air Inc., New York, USA).

It is possible to find the switched dynamical equation for the 7 -mode system by taking the derivative of (5) and substituting the pressure dynamics $\dot{P}_{P}$ and $\dot{P}_{N}$ :

$$
\dddot{y}= \begin{cases}f+\frac{\dot{\tau}_{E x t}}{M} & , \text { mode } M_{1} \\ f+(-1)^{j} g_{j}+\frac{\dot{\tau}_{E x t}}{M} & , \text { mode } M_{j} \neq M_{1}\end{cases}
$$

where the integer $j$ ranges from 2 and 7 and

$$
\begin{array}{cc}
f=\frac{-b_{V}}{M} \ddot{y}-\frac{k}{M}\left(\frac{A_{P} P_{P}}{l / 2+y}+\frac{A_{N} P_{N}}{l / 2-y}\right) \dot{y} \\
g_{2}=\frac{k r T}{M} \frac{Q\left(P_{S}, P_{P}\right)}{(l / 2+y)} & g_{3}=\frac{k r T}{M} \frac{Q\left(P_{P}, P_{E}\right)}{(l / 2+y)} \\
g_{4}=\frac{k r T}{M} \frac{Q\left(P_{N}, P_{E}\right)}{(l / 2-y)} & g_{5}=\frac{k r T}{M} \frac{Q\left(P_{S}, P_{N}\right)}{(l / 2-y)} \\
g_{6}=g_{2}+g_{4} & g_{7}=g_{5}+g_{3}
\end{array}
$$

where $P_{S}$ and $P_{E}$ are the pressures of the compressed air supply and the exhaust (atmosphere), respectively; $l$ is the total length of the chamber, $k$ refers to the polytropic constant, $T$ is the supply temperature, and $r$ refers to the universal gas constant $(\mathrm{J} /(\mathrm{kg} \mathrm{K}))$. In general, $Q\left(P_{U p}, P_{\text {Down }}\right)$, in which $P_{U p}$ is the upstream pressure and $P_{D o w n}$ is the downstream pressure, refers to the expression for the mass flow rate through an orifice. It should be noted that the functions $g_{2}$ through $g_{7}$ are all positive or equal to zero. 


\section{SLIDING-MODE CONTROL of a DUAL PNEUMATIC TELE- OPERATION SYSTEM}

\subsection{Position-Position Control}

For a teleoperation system, the master and slave dynamics will be the same as those described previously with the difference that the common variables will be re-labeled as shown in Table 2. The block diagram in Figure 2 shows the architecture of a position-position bilateral teleoperation system (also called Position Error Based in [3]). In this setup, the human operator dynamics, $Z_{h}$, and the environment dynamics, $Z_{e}$, are unknown or uncertain; $\tau_{h}$ and $\tau_{e}$ are the operator force applied on the master and the environmental force applied on the slave, respectively. The $\tau_{h}^{*}$ and $\tau_{e}^{*}$ variables are the continuous exogenous input forces from the operator and the environment, which have limited energy and as such are bounded.

The equations for the master and slave dynamics are

$$
\begin{array}{r}
M \ddot{y}_{m}=A\left(P_{P, m}-P_{N, m}\right)-b_{V} \dot{y}_{m}+\tau_{h} \\
M \ddot{y}_{s}=A\left(P_{P, s}-P_{N, s}\right)-b_{V} \dot{y}_{s}-\tau_{e}
\end{array}
$$

Substituting the variables from Table 2 into (6) we obtain

$$
\begin{gathered}
\dddot{y}_{m}= \begin{cases}f_{m}+\dot{\tau}_{h} / M & , \text { mode } M_{1} \\
f_{m}+(-1)^{v} g_{v, m}+\dot{\tau}_{h} / M & , \text { mode } M_{v} \neq M_{1}\end{cases} \\
\dddot{y}_{s}= \begin{cases}f_{s}-\dot{\tau}_{e} / M & , \text { mode } M_{1} \\
f_{s}+(-1)^{t} g_{t, s}-\dot{\tau}_{e} / M & , \text { mode } M_{t} \neq M_{1}\end{cases}
\end{gathered}
$$

where $v \in\{1, \ldots, 7\}$ and $t \in\{1, \ldots, 7\}$.

For sliding-mode position control purposes, let us define the switching function as

$$
s_{p}=\frac{\ddot{e}_{p}}{\omega_{p}^{2}}+\frac{2 \xi_{p} \dot{e}_{p}}{\omega_{p}}+e_{p}
$$

in which $e_{p}=y_{m}-y_{s}$ and $\xi$ and $\omega$ are constant and positive numbers. It should be noted that the slave controller uses this switching function $\left(s_{p}\right)$ whereas the master controller uses its negative $\left(-s_{p}\right)$.

Thus, the switching function $s_{p}$ provides a measure of the distance from the sliding surface based on the position, velocity, acceleration errors. Take the derivative of (9) to get

$$
\begin{aligned}
\dot{s}_{p} & =\frac{\dddot{e}_{p}}{\omega^{2}}+\frac{2 \xi \ddot{e}_{p}}{\omega}+\dot{e}_{p} \\
& =\frac{\dddot{y}_{m}-\dddot{y}_{s}}{\omega^{2}}+\frac{2 \xi \ddot{e}_{p}}{\omega}+\dot{e}_{p}
\end{aligned}
$$

Considering the slave actuator (the workflow is symmetric for the master), by substituting the actuator dynamics (6) into (10) through $y_{s}$ we obtain

$$
\dot{s}_{p}= \begin{cases}\lambda_{s} & , \text { mode } M_{1} \\ \lambda_{s}+(-1)^{i} g_{t, s} / \omega^{2} & , \text { mode } M_{t}, \quad(2 \leq i \leq 7)\end{cases}
$$


where $\lambda=\left(f_{s}-\dddot{y}_{m}+\dot{\tau}_{E x t} / M\right) / \omega^{2}+2 \xi \ddot{e}_{p} / \omega+\dot{e}_{p}$ with $\tau_{E x t}=-\tau_{e}$ for the slave or $\tau_{h}$ for the master.

To ensure the convergence to the sliding surface $s_{p}=0$, we wish to have the $\dot{s}_{p}$ such that $s_{p}$ is always approaching zero regardless of its sign. To ensure this, we will invoke the seven different modes of the open-loop system based on the current value of $s_{p}$ relative to five distinct regions for the value of $s_{p}$. These regions of $s_{p}$ and the operating mode of the system as selected by the sliding-mode controller are illustrated in Table 3. This attempts to ensure $\dot{s}_{p}<0$ when $s_{p}>0$ and $\dot{s}_{p}>0$ when $s_{p}<0$, leading to the convergence to $s_{p}=0$. The choice rules concerning modes $M_{3}$ vs $M_{5}$ and $M_{2}$ vs $M_{4}$ are detailed in [7].

When we utilize the pneumatic controller that alters the operating mode of the pneumatic actuator system based on Table 3, for the lowest error band $\left|s_{p}\right|<\epsilon$, we use the mode $M_{1}$ which has no active effect on the system (i.e., no actuation). For the highest positive error band $s_{p}>\beta$, we use the mode $M_{7}$, which exerts the highest drive (causing the highest piston acceleration) in the negative direction. Conversely, for the largest negative error $s_{p} \leq-\beta$, we utilize mode $M_{6}$, which has the highest drive in the positive direction [6]. Evidently, this control action is designed to ensure that the system is always approaching the $s_{p}=0$ surface. For methodology on the selection of $\beta$ and $\epsilon$ please refer to [7].

To be able to analyze the closed-loop position tracking performance, consider the Lyapunov function candidate

$$
V_{l y a}=\frac{1}{2} s_{p}^{2}
$$

which is positive-definite. Therefore, if $\dot{V}_{l y a}<0$, then $V_{l y a}$ will be decreasing. If $V_{l y a}$ is decreasing, $\left|s_{p}\right|$ will also be decreasing. Assuming $s_{p}$ is initially bounded and $\left|s_{p}\right|$ is always decreasing, then $s_{p}$ will always be bounded. This means that $s_{p}$ will approach zero if we control the system so that

$$
\dot{V}_{l y a}=\dot{s}_{p} s_{p}<-\eta_{p}\left|s_{p}\right|
$$

for some positive constant $\eta_{p}>0$ [22]. This condition can be rewritten as

$$
\begin{cases}\dot{s}_{p}>\eta_{p} & \text { if } s_{p}<0 \\ \dot{s}_{p}<-\eta_{p} & \text { if } s_{p}>0\end{cases}
$$

In the above, $\dot{s}_{p}$ is found by taking the derivative of $(9)$ :

$$
\dot{s}_{p}=\frac{\dddot{e}_{p}}{\omega_{p}^{2}}+\frac{2 \xi_{p} \ddot{e}_{p}}{\omega_{p}}+\dot{e}_{p}
$$

Let us consider two possible cases for the sign of $\dot{s}_{p}$ in the following.

$$
-\operatorname{sgn}\left(s_{p}\right) \cdot \dot{s}_{p}>\eta_{p}
$$

Based on the aforementioned sliding-mode control (specifically, see Table 3), the master and slave systems' closed-loop dynamics become

$$
\dddot{y}_{m}=f_{m}-\operatorname{sgn}\left(s_{p}\right) \cdot g_{v, m}+\dot{\tau}_{h} / M \quad v \in\{3,5,7\}
$$




$$
\dddot{y}_{s}=f_{s}+\operatorname{sgn}\left(s_{p}\right) \cdot g_{t, s}-\dot{\tau}_{e} / M \quad t \in\{2,4,6\}
$$

because, as noted earlier, the slave controller uses the switching function $s_{p}$ while the master controller uses the switching function $-s_{p}$. Given

$$
\dddot{e}_{p}=\dddot{y}_{m}-\dddot{y}_{s}=\left(f_{m}-f_{s}\right)-\operatorname{sgn}\left(s_{p}\right) \cdot\left(g_{v, m}+g_{t, s}\right)+\left(\dot{\tau}_{h}+\dot{\tau}_{e}\right) / M
$$

Substituting (18) into (15), we find

$$
\dot{s}_{p}=\lambda_{p}-\operatorname{sgn}\left(s_{p}\right) \cdot\left(g_{v, m}+g_{t, s}\right) / \omega_{p}^{2}
$$

where

$$
\lambda_{p}=\frac{\left(f_{m}-f_{s}\right)+\left(\dot{\tau}_{h}+\dot{\tau}_{e}\right) / M}{\omega_{p}^{2}}+\frac{2 \xi_{p}}{\omega_{p}} \ddot{e}_{p}+\dot{e}_{p}
$$

Finally, by substituting (19) into (16) we find that condition (16) is fulfilled if and only if the following condition is met:

$$
\left(g_{v, m}+g_{t, s}\right)>\left(\eta_{p}+\operatorname{sgn}\left(s_{p}\right) \cdot \lambda_{p}+\right) \omega_{p}^{2}
$$

Therefore, if the positive-valued functions $g_{i, m}$ and $g_{i, s}$ for $i \in\{2, \cdots, 7\}$ are sufficiently large, then the control strategy can satisfy condition (14) (or equivalently (21)) as long as $\lambda_{p}$ is bounded.

On the other hand, since all twelve functions $g_{i, m}$ and $g_{i, s}$ are proportional to $C_{v a l}$, the valve's mass flow rate constant, then choosing a large enough valve will ensure that these scalar functions will be sufficiently large and thus the convergence to the sliding surface $s_{p}=0$ over time is guaranteed.

One of the distinct advantages of the position-position control architecture is that it does not require any force sensors for ensuring master/slave force tracking and thus providing some level of haptic feedback to the operator, but in general it comes at the cost of non-ideal master/slave force tracking [24]. As a remedy, the force-position control in the next section is proposed.

\subsection{Force-Position Control}

The block diagram in Figure 3 shows the architecture for a force-position bilateral teleoperation system (also called Direct Force Reflection in [3]); the slave side of the system is identical to that in the positionposition teleoperation case. This means that the slave actuator controller uses the same switching function (9) as in position-position architecture, and therefore the position tracking performance and stability of the slave subsystem is ensured in the same way as before. This teleoperation architecture is known to provide better transparency than the position-position one but requires two additionnal force sensors.

Force-position control is different from position-position control in that the control action for the master actuator comes from the readings of a sensor measuring slave/environment contact forces. Thus, the master's sliding-mode controller uses a new switching function given by

$$
s_{f}=\frac{\ddot{e}_{f}}{\omega_{f}^{2}}+\frac{2 \xi_{f} \dot{e}_{f}}{\omega_{f}}+e_{f}
$$


where $e_{f}=-\tau_{h}-\tau_{e}$ is the force tracking error between the master and the slave (according to (7) $\tau_{h}$ and $\tau_{e}$ have opposite signs, thus $\left(-\tau_{h}-\tau_{e}\right)$ is the force error). To be able to analyze the force tracking performance, consider the following Lyapunov function candidate

$$
V_{l y b}=\frac{1}{2} s_{f}^{2}
$$

Evidently, $V_{l y b}$ is a positive-definite function and, if $\dot{V}_{l y b}<0, V_{l y b}$ will be decreasing. If $V_{l y b}$ is decreasing, $\left|s_{f}\right|$ will also be decreasing. Assuming $s_{f}$ is initially bounded and $\left|s_{f}\right|$ is decreasing, $s_{f}$ will approach zero. This requires that we control the master robot so that

$$
\dot{V}_{l y b}=\dot{s}_{f} s_{f}<-\eta_{f}\left|s_{f}\right|
$$

for some positive constant $\eta_{f}>0$. This condition can be rewritten as

$$
\begin{cases}\dot{s}_{f}>\eta_{f} & \text { if } s_{f}<0 \\ \dot{s}_{f}<-\eta_{f} & \text { if } s_{f}>0\end{cases}
$$

Take the derivative of (22) to obtain

$$
\dot{s}_{f}=\frac{\dddot{e}_{f}}{\omega_{f}^{2}}+\frac{2 \xi_{f} \ddot{e}_{f}}{\omega_{f}}+\dot{e}_{f}
$$

Let us consider two possible cases for the sign of $\dot{s}_{f}$ in the following.

- Case 1: If we consider that $s_{f}$ is positive, according to (25) we have the following condition

$$
\dot{s}_{f}<-\eta_{f}
$$

Based on the aforementioned sliding-mode control, the master and slave dynamics become

$$
\begin{aligned}
& \dddot{y}_{m}=f_{m}-g_{t, m}+\dot{\tau}_{h} / M \quad t \in\{3,5,7\} \\
& \dddot{y}_{s}=f_{s}+(-1)^{i} g_{i, s}-\dot{\tau}_{e} / M \quad i \in\{1-7\}
\end{aligned}
$$

Substituting (28) into (26), we find

$$
\dot{s}_{f}=\lambda_{f}-g_{t, m} M
$$

where

$$
\lambda_{f}=\left(-\dddot{y}_{m}+f_{m}-(-1)^{i} g_{i, s}+\dddot{y}_{s}-f_{s}\right) M+\frac{\dddot{e}_{f}}{\omega_{f}^{2}}+\frac{2 \xi_{f} \ddot{e}_{f}}{\omega_{f}}
$$

Also, substituting (29) into (27) we find

$$
g_{t, m}>\frac{\lambda_{f}+\eta_{f}}{M}
$$

Therefore, (27) is true if and only if condition (31) is met. 
- Case 2: If we consider that $s_{f}$ is negative, according to (25) we have the following condition

$$
\dot{s}_{f}>\eta_{f}
$$

Based on the aforementioned sliding mode control, the master and slave dynamics become

$$
\begin{aligned}
& \dddot{y}_{m}=f_{m}+g_{v, m}+\dot{\tau}_{h} / M \quad v \in\{2,4,6\} \\
& \dddot{y}_{s}=f_{s}+(-1)^{i} g_{i, s}-\dot{\tau}_{e} / M
\end{aligned}
$$

Substituting (33) into (26) we find

$$
\dot{s}_{f}=\lambda_{f}+g_{v, m} M
$$

Finally, substituting (34) into (32) we find

$$
g_{v, m}>\frac{\eta_{f}-\lambda_{f}}{M}
$$

Therefore, (32) is true if and only if condition (35) is met.

Therefore, if the positive-valued functions $g_{i, m}$ for $i \in\{2, \cdots, 7\}$ are large enough, the control strategy can satisfy condition (25). All six functions $b_{i, m}$ are proportional to $C_{v a l}$, the valve's mass flow rate constant, thus choosing a large enough valve will ensure that these scalar functions will be sufficiently large, and thus, ensure the convergence to the sliding surface $s_{f}=0$ within finite time.

\section{EXPERIMENTAL RESULTS}

The purpose of this section is to test the advantage offered by 7 -mode over 3-mode control in the context of teleoperation control of a pneumatic actuator. We first evaluate the position-position teleoperation architecture where the two robots are position controlled with the position of each robot serving as the reference position for the other robot. We then consider the force-position architecture where the slave robot utilizes position control and the master robot utilizes force control. For both of these architectures, we will compare 3-mode versus 7 -mode in terms of position tracking, force tracking, and valve switching activity.

To test the teleoperation control schemes discussed previously, a quasi-periodic input motion pattern was applied by the operator's hand to the master. This input resembled three cycles of back-and-forth motion with an approximately $10 \mathrm{~mm}$ RMS amplitude when the slave was in free space, followed by approximately two seconds of motion causing contact between the slave and its environment, which was a soft material located $14.5 \mathrm{~mm}$ away from the slave's zero position. This entire motion pattern was

repeated by the human operator three times over a 20 second period. The position and force profiles of the master and the slave robots were measured via position and force sensors. 


\subsection{Experimental Setup}

In this paper, experiments were performed with a pair of 1-DOF pneumatic actuators as the master and the slave - see Figure 4. The low friction cylinders (Airpel model M16D100D) have a $16 \mathrm{~mm}$ diameter and a $100 \mathrm{~mm}$ stroke. The piston and shaft mass for each actuator is approximately $\mathrm{M}=900 \mathrm{~g}$. In terms of sensors, a low-friction linear variable differential transformer (LVDT) is connected to the cylinder in order to measure the linear positions.

The pneumatic solenoid valves (Matrix model GNK821213C3K) used to control the air flow have switching times of approximately $1.3 \mathrm{~ms}$ (opening time) and $0.2 \mathrm{~ms}$ (closing time). With such fast switching times, the on/off valves are appropriate for the purposes of the proposed control. The controller is implemented using a dSPACE board (DS1104), running at a sampling rate of $500 \mathrm{~Hz}$. The double differenciation required for the sliding mode control has been performed with Matlab Simulink Discrete derivative blocks. As numerically differentiating twice produces a considerable amount of numerical noise, we compensated for this by filtering the measured (resp.) position and force error by a 2 nd order low-pass Butterworth filter with a cutoff of (resp.) $70 \mathrm{~Hz}$ and $10 \mathrm{~Hz}$. This reduces this noise to an acceptable level but introduces a small delay which the sliding-mode control successfully compensates for. This sampling rate has been chosen according to the open/close bandwidth of the valves and to enable an acceptable tracking response. The experimental setup has the model parameters listed in Table 4 .

\subsection{Position-Position Teleoperation Control}

In this section, we review the experimental results for the position-position architecture using the sliding mode control design in Section 3.1. For this experiment, the following controller parameters were selected from preliminary experiments: $\omega_{p}=50 \mathrm{rad} / \mathrm{s}, \xi_{p}=0.5, \epsilon_{p}=1 \mathrm{~mm}, \beta=3.4 \mathrm{~mm}$. The position-position scheme relied either on the 3 -mode or the 7 -mode sliding control. The results are depicted in Figure 5. From these results, we can see that there is a $58 \%$ improvement in the RMS error of position tracking with the 7-mode based control compared to the 3-mode based control. We can also see from these results that, in terms of the switching activity of the solenoid valves, there is a $28 \%$ reduction in the 7 -mode based control compared to the 3-mode based control. The reason for this is that, in the 3-mode control, each change of mode involves 2 switch transitions (between columns $M_{1}$ to $M_{6}$ or $M_{7}$ of Table 1 ). In the 7-mode control, however, each change of mode involves either only 1 switch transition (between columns $M_{1}$ to $M_{2}-M_{5}$ or $M_{2}-M_{5}$ to $M_{6}$ or $M_{7}$ of Table 1 ) or 2 (between columns $M_{1}$ to $M_{6}$ or $M_{7}$ of Table 1) switch transitions. As a result, 7-mode control leads to less switching activity.

The force tracking performance is approximately the same for the 3-mode and the 7 -mode control schemes as it is evident from Figure 5 . When the slave is in contact with the environment $\left(\tau_{e} \neq 0\right)$, there is good force tracking as $\tau_{h} \approx \tau_{e}$. However, under the free motion case, (i.e., when $\tau_{e} \approx 0$, because the slave is not in contact with the environment), we see a sizable force feedback of about $\pm 10 \mathrm{~N}$ applied by the master to the operator. 


\subsection{Force-Position Teleoperation Control}

In this section, we review the experimental results for the force-position architecture defined in Section 3.2. For this experiment, the following force controller parameters were selected for the master controller: $\omega_{f}=50 \mathrm{rad} / \mathrm{s}, \xi_{f}=1.0, \epsilon_{f}=0.5 \mathrm{~N}, \beta=1.7 \mathrm{~N}$. The slave controller utilized the same control parameters described in Section 4.2. The force-position scheme was used with both 3-mode and 7-mode sliding control. The results are charted in Figure 6. From these results we can see that there is a $44 \%$ improvement in position tracking error and a $20 \%$ improvement in force tracking error for the 7 -mode control when compared to the 3-mode control under force-position based control. We can also see from these results that, in terms of the switching activity of the solenoid valves, there is a $27 \%$ reduction in the 7-mode based control compared to the 3-mode based control.

\subsection{Performance Comparison with more traditional controllers}

We performed a series of experiments where the proposed sliding mode control was replaced by a PD

controller. The PD gains $\left(K_{p_{p}}\right.$ and $\left.K_{d_{p}}\right)$ were set experimentally so that the system reacts as fast as possible with small tracking errors. Results we obtained are depicted in Figures 7 and 8. It is difficult to compare the performances between 3 and 7 modes as the input of the system is applied by a human and is therefore not exactly reproducible. Nevertheless, it is clear that better performances were obtained with the sliding mode controller (Figure 5 and 6 ) compared to the $\mathrm{P}(\mathrm{D})$ controller. It is noteworthy that we could not further increase more the proportional gain $K_{p_{p}}$ as oscillations appeared while $K_{p_{p}}$ was greater than 0.04. For the force controller, we could not significantly improve the response with a PI controller. This is why we show here only plots with a proportional controller $\left(K_{p_{f}}\right)$ for the force loop.

\section{ANALYSIS and DISCUSSION}

\subsection{Position-Position Teleoperation Control}

In this section, we analyze the experimental results for the position-position architecture reported in Section 4.2 and depicted in Figure 5. From these results, we can observe reasonable position tracking. This architecture also demonstrates good force tracking when the slave is in contact with the environment. However, under the free-motion case (i.e., $\tau_{e} \approx 0$ ), while the slave is not in contact with the environment, we see a sizable and varying force feedback of about $\pm 10 \mathrm{~N}$ in magnitude applied by the master to the operator. This severely disrupts the perception of free motion for the operator and is undesirable. In the following, we try to understand the cause of this unwanted force feedback to the operator.

To illuminate the reason for the large force feedback of about $\pm 10 \mathrm{~N}$ experienced by the operator when the slave is in free space, let us examine the sum of forces acting on each actuator. The dynamics 
of the master and the slave according to (5) are

$$
\begin{gathered}
M \ddot{y}_{s}=-\tau_{e}+\tau_{s}-\tau_{F, s} \\
M \ddot{y}_{m}=\tau_{h}+\tau_{m}-\tau_{F, m}
\end{gathered}
$$

where $\tau_{s}=\left(A_{P} P_{P, s}-A_{N} P_{N, s}\right), \tau_{m}=\left(A_{P} P_{P, m}-A_{N} P_{N, m}\right), \tau_{F, s}=b_{V} \dot{y}_{s}$ is the slave viscous friction force, and $\tau_{F, m}=b_{V} \dot{y}_{m}$ is the master viscous friction force. In fact, in Figure $2, \tau_{s}$ and $\tau_{m}$ are the inputs to the two Actuator Dynamics blocks, and we have $\tau_{s}=-\tau_{m}$ because $\tau_{s}$ is implemented based on the switching function $s_{p}$ while $\tau_{m}$ is implemented based on the switching function $-s_{p}$. First, consider the slave actuator in the free motion case where $\tau_{e}=0$. We have

$$
\tau_{s}=M \ddot{y}_{s}+\tau_{F, s}
$$

As mentioned above, in position-position control of Figure 2, the master's control action is in the opposite direction to that of the slave:

$$
\tau_{m}=-\tau_{s}
$$

Substituting (38) into (39) gives us

$$
\tau_{m}=-M \ddot{y}_{s}-\tau_{F, s}
$$

Substituting (40) into (37) and solving for $\tau_{h}$ leads to

$$
\tau_{h}=M\left(\ddot{y}_{m}+\ddot{y}_{s}\right)+\tau_{F, m}+\tau_{F, s}
$$

Therefore, the operator has to overcome the viscous friction and the inertias of both the master and the slave actuators in order to accelerate the master - this is a serious deficiency of position-position control especially in pneumatic systems where the viscous friction is high.

In our experiments, the peak accelerations are low $\left(\ddot{y}_{m} \approx \dot{y}_{s}=0.8 \mathrm{~m} / \mathrm{s}^{2}\right)$ and the mass $M=0.1 \mathrm{~kg}$, so inertia effect is around $8 \cdot 10^{-2} \mathrm{~N}$. Peak velocity is $\dot{y}_{m} \approx \dot{y}_{s}=100 \mathrm{~mm} / \mathrm{s}$ and $b_{V}=50 \mathrm{~N} \mathrm{~s} / \mathrm{m}$. Therefore, the peak of the viscous friction force will be $\tau_{F, m} \approx \tau_{F, s}=5 \mathrm{~N}$ or a total unwanted force of $\tau_{F, m}+\tau_{F, s} \approx 10 \mathrm{~N}$. Therefore, it is the viscous friction that is causing a heavy loading in terms of the haptic feedback to the operator when the slave is in free space.

To further evaluate this phenomenon, we simulated in Simulink the free motion case. The results of this simulation are shown in Figure 9. These results also demonstrate a $\pm 10 \mathrm{~N}$ force, which corroborates the analytical and experimental results that we have obtained.

For the case where the slave actuator is in contact with the environment $\left(\tau_{e} \neq 0\right)$, the hand was stopped by the force feedback and, as a result, the hand velocity decreased to near zero. Since the unwanted force is caused by the viscous friction, which is proportional to the actuators velocity, in Figure 5 the unwanted force vanished as the master and slave velocities went towards zero. 


\section{$5.2 \quad$ Force-Position Teleoperation Control}

In this section, we analyze the experimental results for the force-position architecture reported in Section 4.3 and depicted in Figure 6.

From these results, we can observe that during both contact motion and free motion cases, there is good force tracking $\left(-\tau_{h} \approx \tau_{e}\right)$ as well as good position tracking. The force-position control scheme with pneumatic actuators does not have the sizable force feedback in free-motion that was observed in the position-position control scheme.

The force controller on the master side minimizes the difference in measured force between the slave and master side. As a result, the controller on the master side compensates for the force of friction on the master actuator. The force measured by the slave actuator does not include the force of friction on the slave side. Therefore, the only feedback felt by the hand through the haptic interface is the environmental force measured on the slave actuator.

This makes the force-position teleoperation architecture more advantageous then the position-position teleoperation architecture in terms of free motion force tracking. Although the force-position had better force tracking, it was observed that the position-position control architecture had a $16 \%$ improvement in position tracking when compared to the force-position control architecture.

\section{CONCLUDING REMARKS}

We described a pneumatic open-loop actuator system with seven modes of operation based on the state of the on/off solenoid valves. For such a pneumatic system, sliding-mode control laws were developed for position and force control. The sliding control laws were utilized in two different teleoperation architectures: position-position and force-position. The sliding controllers for the pneumatic system selects one of these seven modes of operation at any given time based on the magnitudes and signs of the switching functions $s_{p}$ and $s_{f}$ for the position and force controllers, respectively.

These closed-loop controls were experimentally verified on a setup consisting of a pair of symmetric pneumatic actuators. For comparison, the experiments were conducted for both the new 7 -mode control and the traditional 3-mode control. It was demonstrated that, for both teleoperation architectures, there was a 44\%-58\% improvement in position tracking error and up to $27 \%$ reduction in the switching activity with the 7-mode controller when compared to the 3-mode controller. It was also demonstrated that there was a $20 \%$ improvement in force tracking error for the 7 -mode controller in the force-position architecture when compared to the 3-mode controller in the same architecture.

In the position-position architecture, it was found that dynamic friction forces were causing large force applications $( \pm 10 \mathrm{~N})$ on the operator hand under the free motion case $\left(\tau_{e}=0\right)$. Thus, in terms of force tracking error, the force-position architecture is preferable for pneumatic actuators. We can see in figure 5 that the free-motion input impedance displayed to the operator was greatly reduced for the force-position architecture compared to the position-position architecture. The 7-mode based 
teleoperation control has performed well in the experiments. This controller would be a viable choice for use whenever a teleoperated robot uses on/off valves for actuation of the pneumatic chamber.

As written above, one of the main drawbacks of this system is the sound produced by the solenoid valves. However, this phenomenon could be diminished by putting the valves away from the cylinders. Unfortunately, the length of the hoses between valves and cylinders introduces an air propagation delay. Having studied the positive effects of 7 -mode control (compared to 3-mode control) on positioning accuracy and switching activity in a teleoperation system, the next step would be to examine the effect of this time delay on this system.

In this paper, we had neglected the time delays. In general, time delay may destabilize an otherwise stable system. As has been demonstrated in [17], a regular sliding-mode controller with some modification can control a slave system to perform a task well independently of time delay. Therefore, future research can explore using sliding-mode control to handle time delays in a pneumatic system.

\section{REFERENCES}

[1] Kyoungkwan Ahn and Shinichi Yokota. Intelligent switching control of pneumatic actuator using on/off solenoid valves. Mechatronics, 15(6):683-702, 2005.

[2] Robert O. Ambrose, Hal Aldridge, R. Scott Askew, Robert R. Burridge, William Bluethmann, Myron Diftler, Chris Lovchik, Darby Magruder, and Fredrik Rehnmark. Robonaut: Nasa's space humanoid. Humanoid Robotics IEEE Intelligent Systems, 15(4):57-63, July/August 2000.

[3] A. Aziminejad, M. Tavakoli, R.V. Patel, and M. Moallem. Transparent time-delayed bilateral teleoperation using wave variables. Control Systems Technology, IEEE Transactions on, 16(3):548 -555 , may 2008.

[4] Peter Beater. Pneumatic Drives - System Design, Modelling and Control. Springer, Berlin, 2006.

[5] Alexis Girin, Franck Plestan, Xavier Brun, and Alain Glumineau. High-order sliding-mode controllers of an electropneumatic actuator: Application to an aeronautic benchmark. International Journal of Control, 79(2):119-131, 2006.

[6] Sean Hodgson, Minh Quyen Le, Mahdi Tavakoli, and Minh Tu Pham. Sliding-mode control of nonlinear discrete-input pneumatic actuators. In Proceedings of IEEE/RSJ International Conference on Intelligent Robots and Systems (IROS), pages 738-743, San Francisco, USA, September 2011.

[7] Sean Hodgson, Minh Quyen Le, Mahdi Tavakoli, and Minh Tu Pham. Improved tracking and switching performance of an electro-pneumatic positioning system. Mechatronics, 22(1):1 - 12, 2012. 
[8] Asier Ibeas and Manuel de la Sen. Robustly stable adaptive control of a tandem of master-slave robotic manipulators with force reflection by using a multiestimation scheme. IEEE Transactions on Systems, Man, and Cybernetics - Part B, 36(5):1162-1179, 2006.

[9] Farrokh Janabi-Sharifi and Iraj Hassanzadeh. Experimental analysis of mobile-robot teleoperation via shared impedance control. IEEE Transactions on Systems, Man, and Cybernetics - Part B, 41(2):591-606, 2011.

[10] Daniel Labonte, Patrick Boissy, and François Michaud. Comparative analysis of 3-d robot teleoperation interfaces with novice users. IEEE Transactions on Systems, Man, and Cybernetics - Part B, 40(5):1331-1342, 2010.

[11] Minh Quyen Le, Minh Tu Pham, Richard Moreau, and Tanneguy Redarce. Transparency of a pneumatic teleoperation system using on/off solenoid valves. In Proceedings of 2010 19th IEEE International Symposium on Robot and Human Interactive Communication, pages 15-20, Viareggio, Italy, September 2010.

[12] Minh Quyen Le, Minh Tu Pham, Mahdi Tavakoli, and Richard Moreau. An enhanced sliding-mode control for a pneumatic-actuated teleoperation system. In Proceedings of IEEE/RSJ International Conference on Intelligent Robots and Systems (IROS), pages 659-664, San Francisco, USA, September 2011.

[13] Minh Quyen Le, Minh Tu Pham, Mahdi Tavakoli, and Richard Moreau. Sliding mode control of a pneumatic haptic teleoperation system with on/off solenoid valves. In Proceedings of 2011 IEEE International Conference on Robotics and Automation (ICRA), Shanghai, China, May 2011.

[14] Han Koo Lee, Gi Sang Choi, and Gi Heung Choi. A study on tracking position control of pneumatic actuators. Mechatronics, 12(6):813-831, 2002.

[15] Arcangelo Messina, Nicola Ivan Giannoccaro, and Angelo Gentile. Experimenting and modelling the dynamics of pneumatic actuators controlled by the pulse width modulation (PWM) technique. Mechatronics, 15(7):859-881, 2005.

[16] T. Nguyen, J. Leavitt, F. Jabbari, and J. E. Bobrow. Accurate sliding-mode control of pneumatic systems using low-cost solenoid valves. IEEE/ASME Transactions on Mechatronics, 12(2):216-219, 2007.

[17] Jong Hyeon Park and Hyun Chul Cho. Sliding-mode controller for bilateral teleoperation with varying time delay. In Proceedings of IEEE/ASME International Conference on Advanced Intelligent Mechatronics, pages 311-316, Atlanta, USA, September 1999.

[18] Stephane Ross, Ryan Waliany, and Matt Swanson. Inverse kinematics and balance control for intuitive teleoperation of a six-legged mining robot. Technical report, Robotics Institute, Carnegie Mellon University, May 2009. 
[19] José M. Sabater, Miguel Hernandez, Roque J. Saltarén, Rafael Aracil, Eugenio Yime, and José M. Azorín. Teleoperated parallel climbing robots in nuclear installations. Industrial Robot: An International Journal, 33(5):381-386, September 2006.

[20] Xiangrong Shen, Jianlong Zhang, Eric J. Barth, and Michael Goldfarb. Nonlinear model-based control of pulse width modulated pneumatic servo systems. Journal of Dynamic Systems, Measurement, and Control, 128:663-669, 2006.

[21] Ming-Chang Shih and Ming-An Ma. Position control of a pneumatic cylinder using fuzzy PWM control method. Mechatronics, 8(3):241-253, 1998.

[22] Jean-Jacques E. Slotine and Weiping Li. Sliding control. In Applied Nonlinear Control, pages 276-310. Prentice Hall, Englewood Cliffs, New Jersey 07632, 1991.

[23] Mostafa Taghizadeh, Ali Ghaffari, and Farid Najafi. Improving dynamic performances of PWMdriven servo-pneumatic systems via a novel pneumatic circuit. ISA Transactions, 48(4):512-518, 2009.

[24] M. Tavakoli, A. Aziminejad, R.V. Patel, and M. Moallem. High-fidelity bilateral teleoperation systems and the effect of multimodal haptics. IEEE Transactions on Systems, Man and Cybernetics - Part B, 37(6):1512-1528, December 2007.

[25] K. Xing, J. Huang, Y. Wang, J. Wu, Q. Xu, and J. He. Tracking control of pneumatic artificial muscle actuators based on sliding mode and non-linear disturbance observer. IET Control Theory and Applications, 4(10):2058-2070, 2010. 


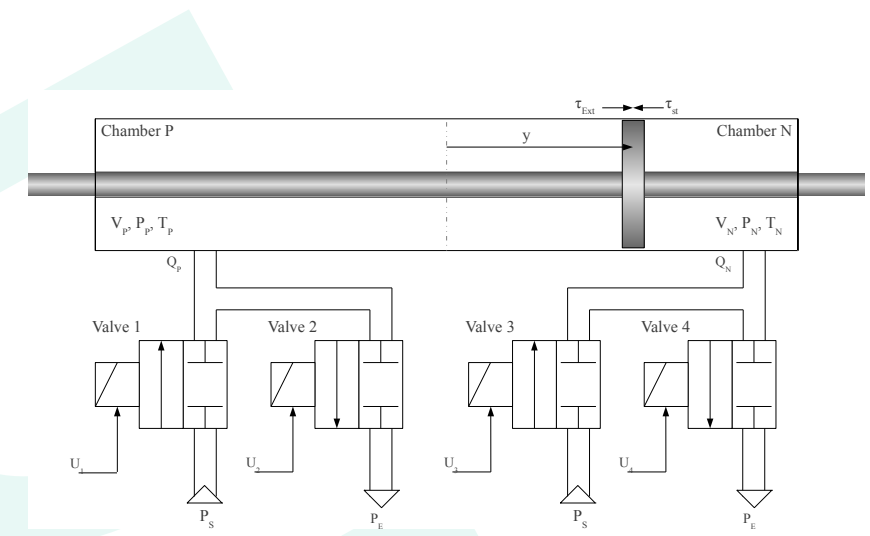

Figure 1: Single pneumatic actuator with 4 on/off solenoid valves

Table 1: Nine Discrete Modes of The Open-Loop Actuator

\begin{tabular}{cccccccc|cc}
\hline \hline & $M_{1}$ & $M_{2}$ & $M_{3}$ & $M_{4}$ & $M_{5}$ & $M_{6}$ & $M_{7}$ & $M_{8}$ & $M_{9}$ \\
\hline$U_{1}$ & 0 & 1 & 0 & 0 & 0 & 1 & 0 & 0 & 1 \\
\hline$U_{2}$ & 0 & 0 & 1 & 0 & 0 & 0 & 1 & 1 & 0 \\
\hline$U_{3}$ & 0 & 0 & 0 & 0 & 1 & 0 & 1 & 0 & 1 \\
\hline$U_{4}$ & 0 & 0 & 0 & 1 & 0 & 1 & 0 & 1 & 0 \\
\hline \hline
\end{tabular}

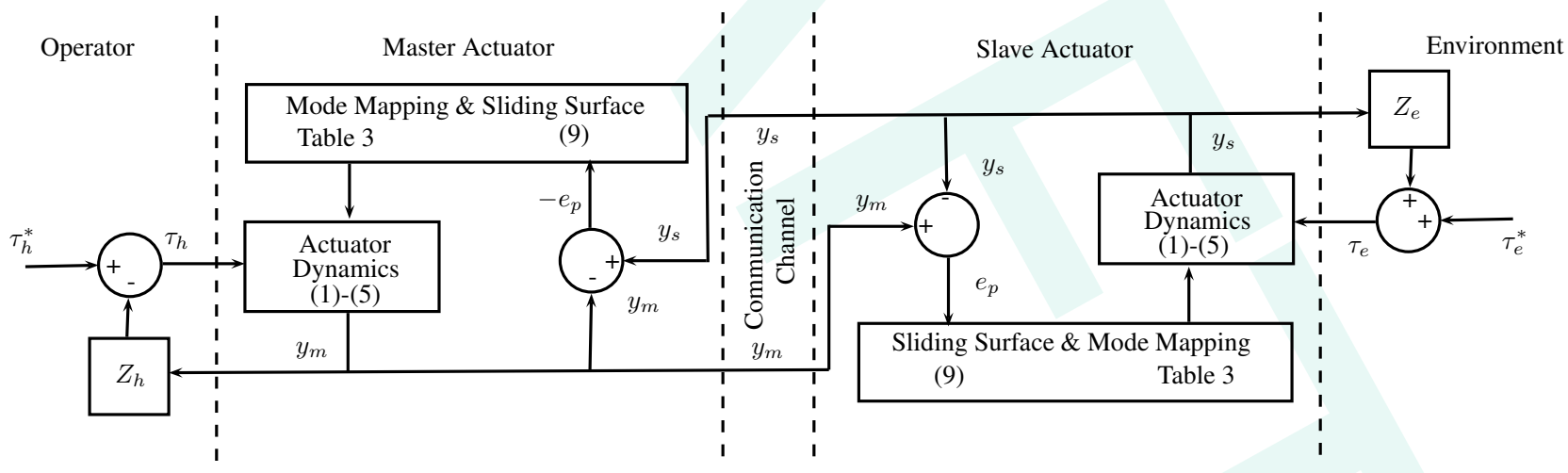

Figure 2: Position-position based teleoperation.

Table 2: Master/slave actuator variable names where $q \in\{P, N\}, v \in\{1, \ldots, 7\}$, and $t \in\{1, \ldots, 7\}$.

\begin{tabular}{|c|c|c|c|c|c|}
\hline Single & $y$ & $P_{q}$ & $\tau_{E x t}$ & $g_{i}$ & $f$ \\
\hline Master & $y_{m}$ & $P_{q, m}$ & $\tau_{h}$ & $g_{v, m}$ & $f_{m}$ \\
\hline Slave & $y_{s}$ & $P_{q, s}$ & $-\tau_{e}$ & $g_{t, s}$ & $f_{s}$ \\
\hline
\end{tabular}


Table 3: Selection of the operating mode based on the value of $s_{p}$.

\begin{tabular}{ccc}
\hline \hline Region of $s_{p}$ & $\begin{array}{c}\text { Selected } \\
\text { operating mode }\end{array}$ & $\begin{array}{c}\text { Magnitude of resulting } \\
\dot{s}_{p} \text { from (11) }\end{array}$ \\
\hline$s_{p}>\beta$ & $M_{7}$ & Large negative \\
$\beta \geq s_{p}>\epsilon$ & $M_{3}$ and $M_{5}$ & Medium negative \\
$\epsilon \geq s_{p}>-\epsilon$ & $M_{1}$ & Small \\
$-\epsilon \geq s_{p}>-\beta$ & $M_{2}$ and $M_{4}$ & Medium positive \\
$-\beta \geq s_{p}$ & $M_{6}$ & Large positive \\
\hline \hline
\end{tabular}

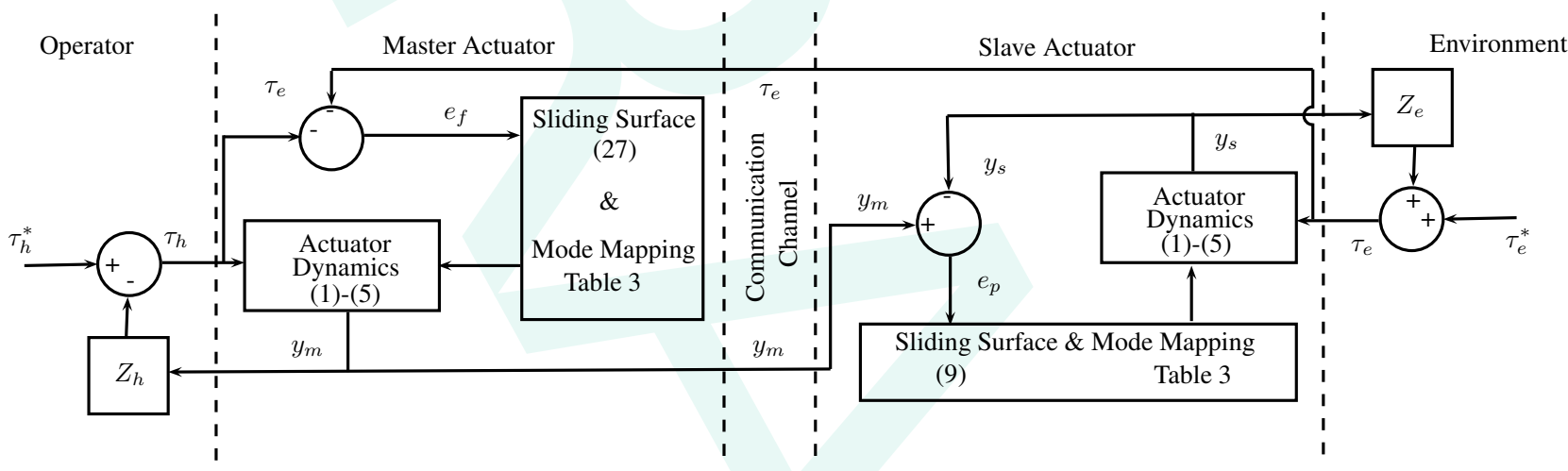

Figure 3: Force-position based teleoperation.

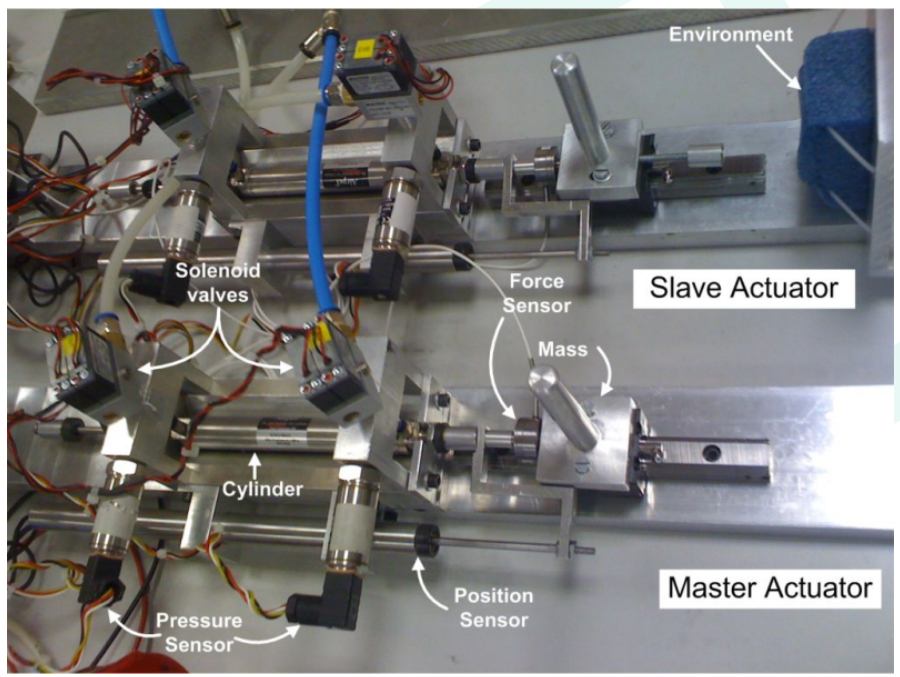

Figure 4: Experimental setup. 
Table 4: System's model parameters.

\begin{tabular}{|c|c|c|}
\hline Var. & Value & Label \\
\hline$l$ & $0.1 \mathrm{~m}$ & Chamber Length \\
\hline$T$ & $296 \mathrm{~K}$ & Supply Temperature \\
\hline$C_{v a l}$ & $3.4 \times 10^{-9} \mathrm{~kg} /(\mathrm{s} \mathrm{Pa})$ & Mass Flow Rate Const. \\
\hline$r$ & $287.0 \mathrm{~J} /(\mathrm{kg} \mathrm{K})$ & Gas Constant \\
\hline$P_{S}$ & $300,000 \mathrm{~Pa}$ & Supply Air Pressure \\
\hline$P_{E}$ & $100,000 \mathrm{~Pa}$ & Exhaust Air Pressure \\
\hline$k$ & 1.2 & Polytropic Constant \\
\hline$A_{P}, A_{N}$ & $1.814 \mathrm{~cm}{ }^{2}$ & Piston Cylinder Area \\
\hline$b_{V}$ & $50 \mathrm{~N} \mathrm{~s} / \mathrm{m}$ & Viscosity Coefficient \\
\hline$M$ & $0.9 \mathrm{~kg}$ & Total Mass of load \\
\hline
\end{tabular}
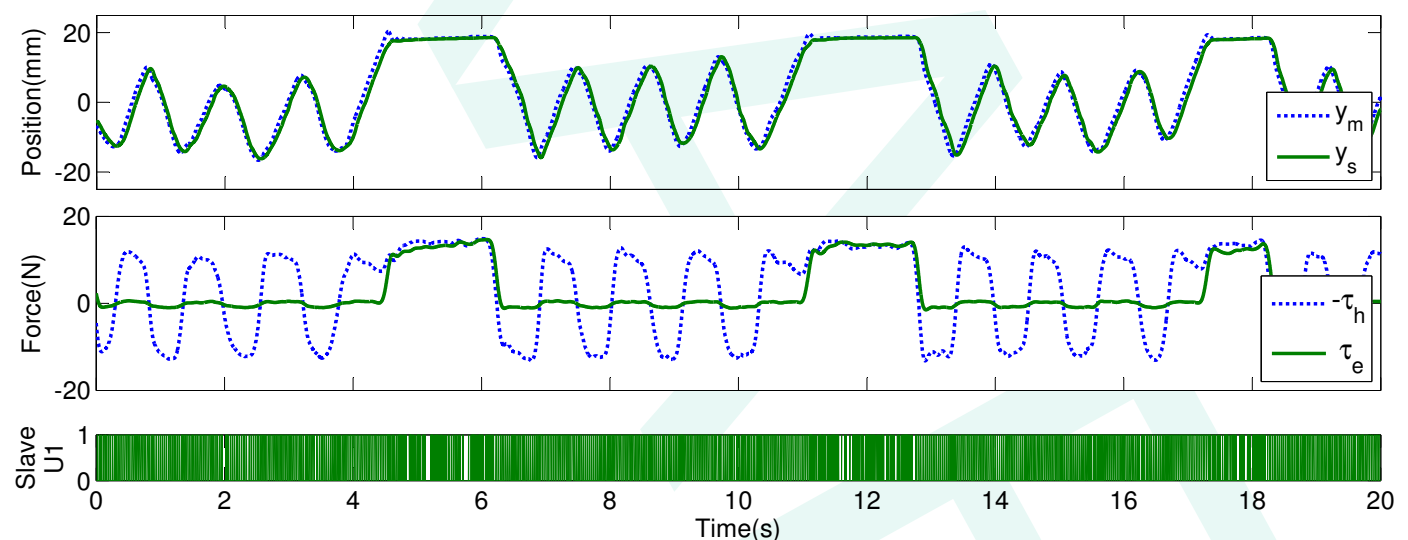

(a)
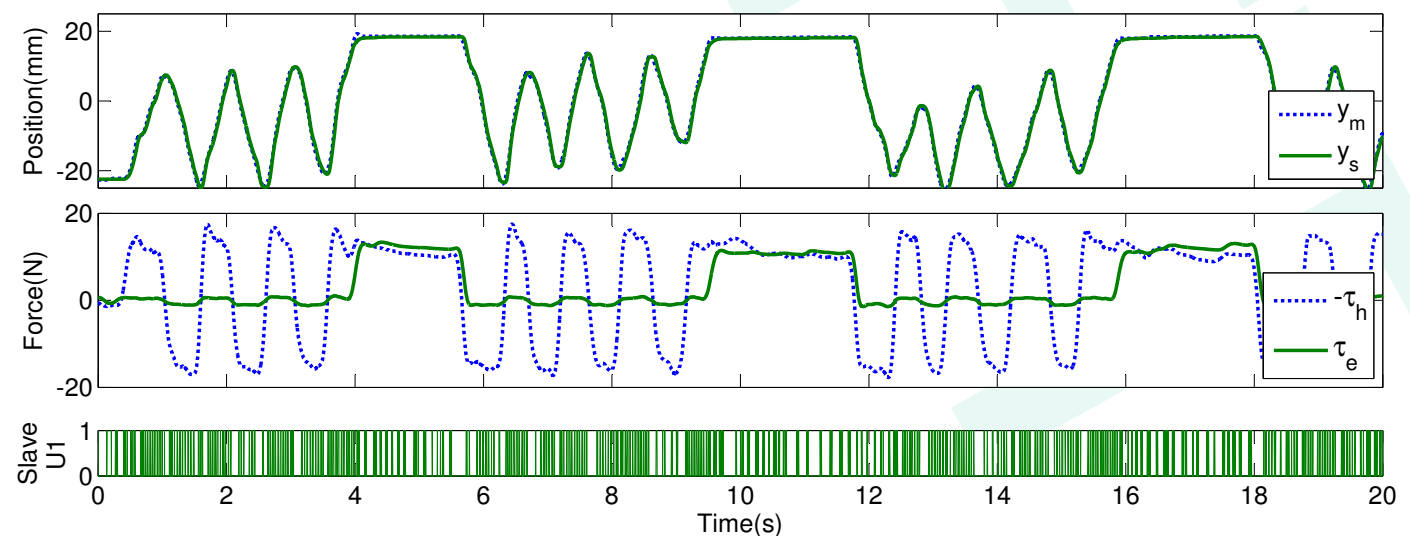

(b)

Figure 5: Position and force tracking profiles for the master and the slave robots in position-position architecture: (a) with 3-Mode sliding control and (b) with 7-Mode sliding control 

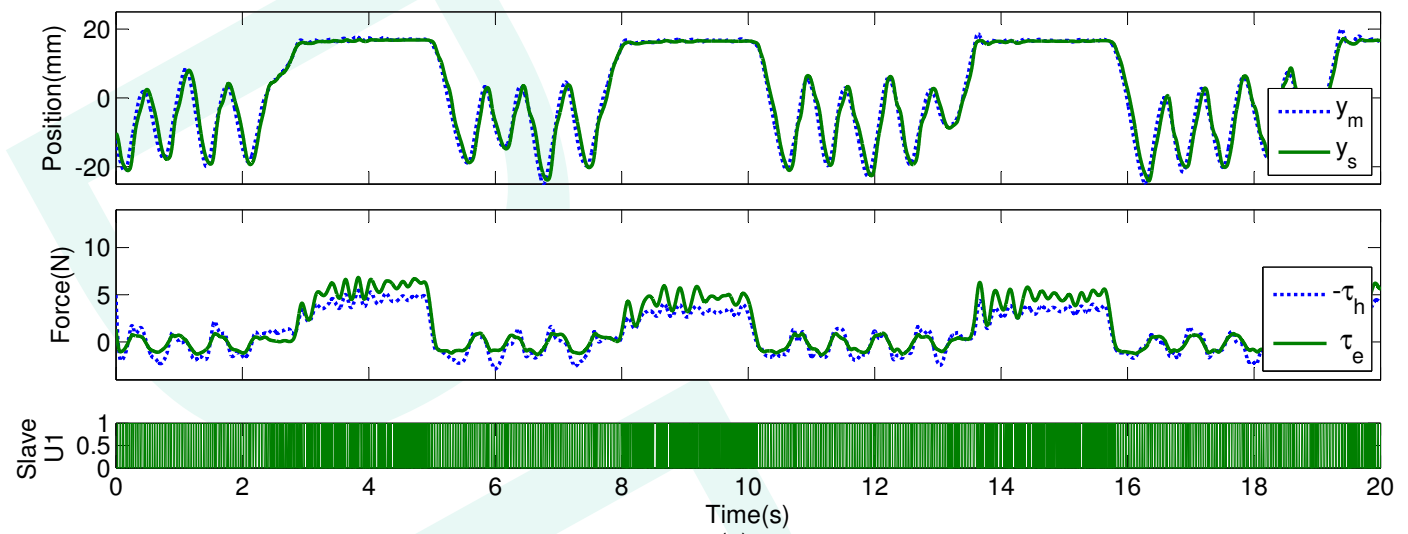

(a)
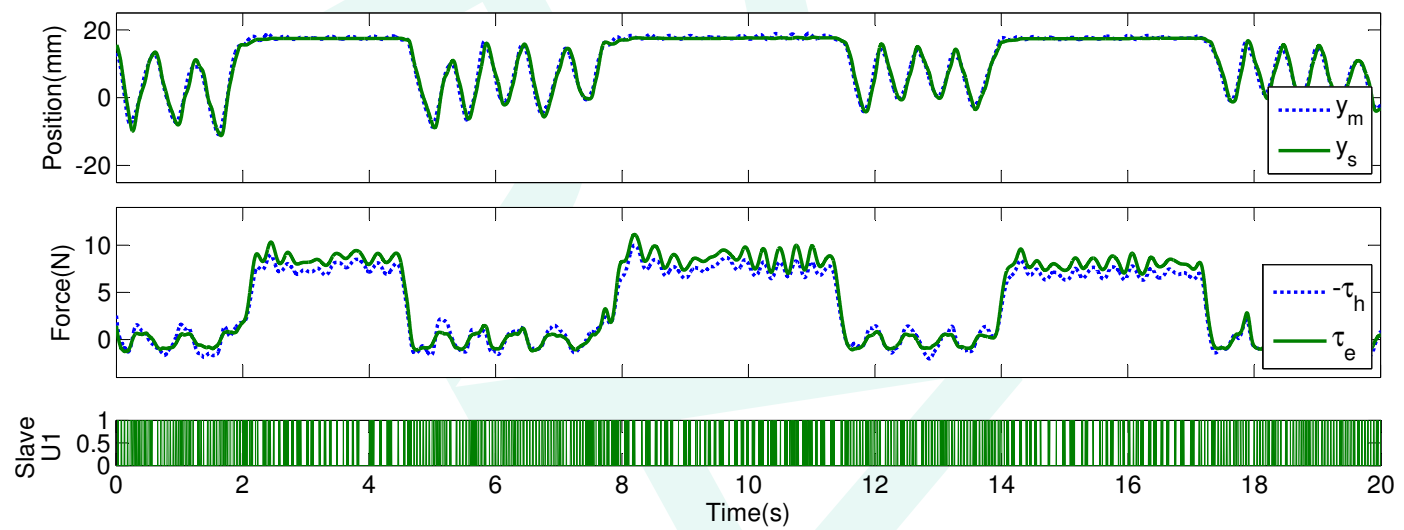

(b)

Figure 6: Position and force tracking profiles for the master and the slave robots in force-position architecture: (a) with 3-Mode sliding control and (b) with 7-Mode sliding control.
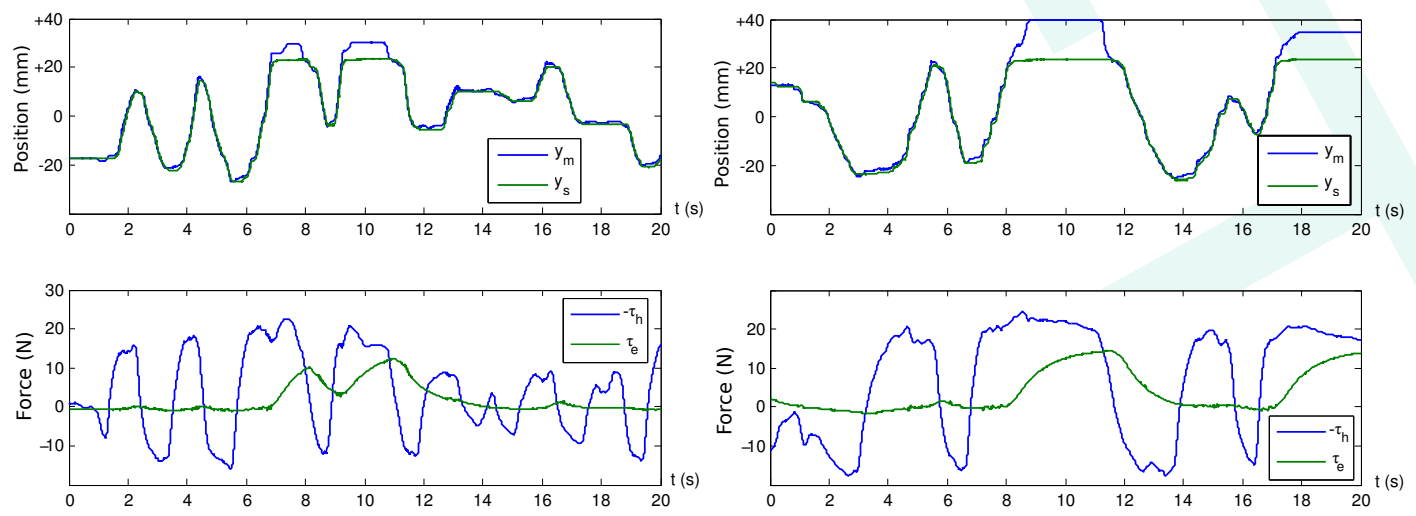

Figure 7: Position-position scheme with PD controller: $K_{p_{p}}=0.04, K_{d}=2.10^{-3}, 3$ mode (left) and 7 mode (right) 

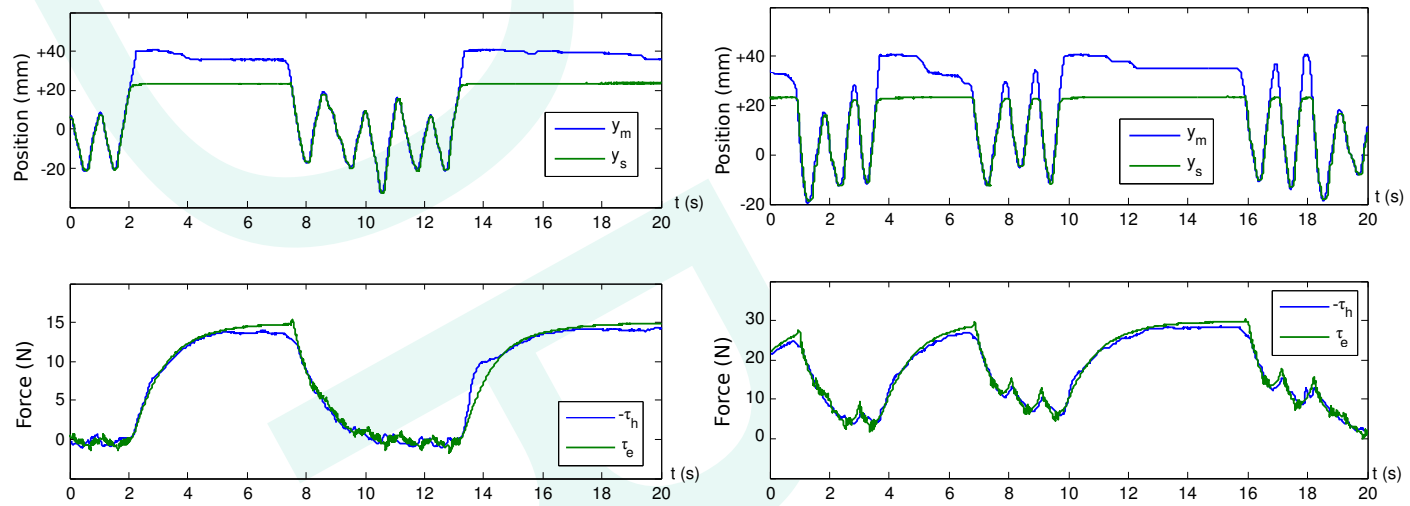

Figure 8: Force-Position scheme with PD controller: $K_{p_{p}}=0.04, K_{d_{p}}=2.10^{-3}, K_{p_{f}}=4.10^{-4}, 3$ mode (left) and 7 mode (right)

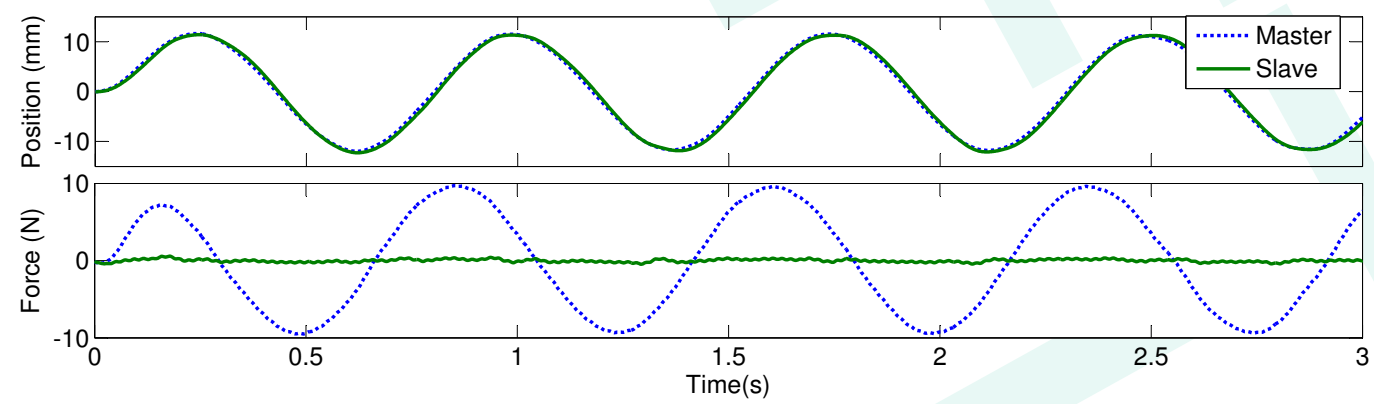

Figure 9: Position-position 7-mode simulated results. 